DOI:

УДК 517.928

М.О. Рашевський, кандидат фіз.-мат. наук, доцент, mora290466@gmail.com

Криворізький національний університет, м. Кривий Ріг

\title{
АСИМПТОТИЧНЕ РОЗВ'ЯЗУВАННЯ ЗАДАЧІ ОПТИМАЛЬНОГО КЕРУВАННЯ НЕСТАЦОНАРНИМИ СИСТЕМАМИ
}

Побудовано асимптотичні розв'язки задачі оптимального керування системами з повільно змінними коефіцієнтами. Досліджено випадок наявності у системі точок повороту. Дано оцінку точності побудованого наближеного розв'язку.

Ключові слова: система оптимального керування; асимптотичний розв'язок; точка повороту.

Asymptotic solutions of systems for automatic control with slowly variable parameters are constructed. The cases of available of turning points in the system are investigated. The estimation of the error of the constructed approximate solution is given.

Keywords: system of automatic control; asymptotic solution; turning point.

\section{Постановка проблеми}

Система оптимального керування, що описується системою диференціальних рівнянь

$$
\frac{d \mathbf{x}(\tau, \varepsilon)}{d t}=\mathbf{A}(\tau, \varepsilon) \mathbf{x}(\tau, \varepsilon)+\mathbf{B}(\tau, \varepsilon) \mathbf{u}(\tau, \varepsilon),
$$

неодноразово досліджувались $[1,4,6]$ у різних припущеннях про іiі коефіцієнти. Тут х $(\tau, \varepsilon)-$ невідомий $n$ - вимірний вектор, $\mathbf{A}(\tau, \varepsilon)$ та $\mathbf{B}(\tau, \varepsilon)$ - відповідно $n \times n$ та $n \times r$ - матриці динамічних коефіцієнтів системи, що $\epsilon$ функціями повільного часу $\tau=\varepsilon t, \tau \in[0 ; L], L<+\infty, \varepsilon>0-$ дійсний малий параметр; $\mathbf{u}(\tau, \varepsilon)-r$ - вимірний вектор керування. Наступну задачу теорії оптимального керування називають задачею оптимальної корекції [4, стор. 370].

Необхідно вибрати керування $\mathbf{u}(\tau)$, що переводить систему (1) із початкового стану (точки $\mathbf{x}_{0} n$ - вимірного простору)

у точку

$$
\mathbf{x}(0, \varepsilon)=\mathbf{x}_{0}
$$

$$
\mathbf{x}(T, \varepsilon)=\mathbf{x}_{T}
$$

за фіксований час $T$, забезпечуючи при цьому мінімум функціоналу

$$
\left.I=\int_{0}^{T}((\mathbf{W}(\tau, \varepsilon) \mathbf{x} \cdot \mathbf{x})+\mathbf{C}(\tau, \varepsilon) \mathbf{u} \cdot \mathbf{u})\right) d t
$$

де $\mathbf{W}(\tau, \varepsilon)$ та $\mathbf{C}(\tau, \varepsilon)$ - додатно означені матриці.

\section{Аналіз останніх досліджень та публікацій}

Сформульована задача вивчалася [4, стор. 370; 6, стор. 84] у зв'язку із практичними застосуваннями у технічних та економічних системах, зокрема у задачі корекції орбіт штучних супутників, задачі керування в економічних системах тощо.

Припускалося, що матриці $\mathbf{A}(\tau, \varepsilon), \mathbf{B}(\tau, \varepsilon), \mathbf{W}(\tau, \varepsilon)$ та $\mathbf{C}(\tau, \varepsilon)$ зображуються асимптотичними рядами вигляду

$$
\mathbf{A}(\tau, \varepsilon)=\sum_{k=0}^{\infty} \varepsilon^{k} \mathbf{A}_{k}(\tau), \mathbf{B}(\tau, \varepsilon)=\sum_{k=0}^{\infty} \varepsilon^{k} \mathbf{B}_{k}(\tau), \mathbf{W}(\tau, \varepsilon)=\sum_{k=0}^{\infty} \varepsilon^{k} \mathbf{W}_{k}(\tau), \mathbf{C}(\tau, \varepsilon)=\sum_{k=0}^{\infty} \varepsilon^{k} \mathbf{C}_{k}(\tau)
$$

При цьому вимагалося, щоб спектр матриці $\mathbf{A}_{0}(\tau)$ був стабільним на досліджуваному проміжку, тобто власні значення згаданої матриці не дорівнювали нулю в окремих його точках і не збігалися в ізольованих точках. Точка досліджуваного проміжку, де будь-який корінь дорівнює нулю, називають точкою нестабільності спектру [3], якщо два або більше коренів дорівнюють нулю (або перетинаються), то говориться про точки повороту $[2 ; 3 ; 5 ; 7 ; 8]$; інші назви - перехідні точки, точки звороту. Останні не є особливими точками для розв'язку системи, а лише для 
його асимптотичного зображення. Тому проблема точок повороту у теорії асимптотичного інтегрування систем розроблялася упродовж минулого століття $[3 ; 7 ; 8]$, найбільш інтенсивно у 60-х роках, і не втратила актуальності в нинішньому столітті. Саме цій проблемі присвячені ряд робіт В.П. Маслова, А.М. Самойленка, М.О. Перестюка, П.Ф. Самусенка, А.І. Шафаревича, В.М. Бобочка, Г.В. Завізіона, І.Г. Ключник та інших авторів. Вивчення систем керування із нестабільним спектром розпочалося роботою [2]. Хоча спектральні особливості, як зазначалося, $\epsilon$ лише особливостями для побудови асимптотичного зображення розв'язку системи рівнянь, проте цікаві ефекти виникають у випадку нестаціонарних систем із нестабільним спектром. Саме ці дослідження і представлені у статті.

\section{Формулювання мети дослідження}

Метою дослідження є побудова асимптотичного розв'язку системи (1) при умовах (2)(4), тобто задачі оптимальної корекції. Система (1) $є$ системою рівнянь 3 повільно змінними коефіцієнтами, тому, як правило, не зводиться до квадратур. Для побудови ії розв'язку використовуються різні наближені методи. Одні з таких методів - асимптотичні, в яких будується наближення точного розв'язку за степенями малого параметра $\varepsilon$. Асимптотичні розв'язки системи (1) побудовано у роботах $[4 ; 6]$ у припущенні, що корені характеристичного рівняння $P(\lambda, \tau) \equiv \operatorname{det}\|\mathbf{A}(\tau)-\lambda \mathbf{E}\|=0$ зберігають постійну кратність на проміжку $[0 ; L] ; \mathbf{E}-$ одинична матриця. Випадок простих коренів полінома $\mathrm{P}(\lambda, \tau)$ розглянуто в [4], а в роботі [6] припускалось, що корені $є$ тотожно кратними. При такій постановці задачі головну роль відіграє спектр не матриці $\mathbf{A}(\tau)$, а записаної далі матриці $\mathbf{D}_{0}(\tau)$. У роботі будується асимптотичне зображення розв'язку задачі за умови нестабільного спектру головної матриці системи.

\section{Виклад основного матеріалу}

Надалі вимагатимемо виконання таких умов.

$1^{0}$. Матриці $\mathbf{A}_{k}(\tau), \mathbf{B}_{k}(\tau), \mathbf{W}_{k}(\tau), \mathbf{C}_{k}(\tau) \epsilon$ нескінченно диференційовними на сегменті $[0 ; L]$; $k \geq 0$.

$2^{0}$. Корені $\lambda_{1}(\tau), \lambda_{2}(\tau), \ldots, \lambda_{n}(\tau)$ рівняння $P(\lambda, \tau)=0 €$ різними на $(0 ; L]$ і принаймні два збігаються при $\tau=0$.

Застосовуючи принцип максимума Понтрягіна до задачі (1), (2), введемо до розгляду гамільтоніан

$$
H=(\mathbf{A}(\tau, \varepsilon) x, \psi)+(\mathbf{B}(\tau, \varepsilon) \mathbf{u}, \psi)-(\mathbf{C}(\tau, \varepsilon) \mathbf{u}, \mathbf{u})-(\mathbf{W}(\tau, \varepsilon) x, x),
$$

де $\psi-n-$ вимірний вектор спряжених змінних.

Згідно з [4; 7] функція

$$
\mathbf{u}^{0}(\tau, \varepsilon)=\frac{1}{2} \mathbf{C}^{-1}(\tau, \varepsilon) \cdot \mathbf{B}^{t}(\tau, \varepsilon) \cdot \psi
$$

надає гамільтоніану максимального значення, отже ії побудова $є$ задачею дослідження; тут і далі значком ${ }^{t}$ позначено транспонування. Розглянемо $2 n$ - вимірний вектор $\mathrm{y}(\tau, \varepsilon)=(\mathrm{x}, \psi)^{t} .3$ урахуванням виразу для $\mathrm{u}^{0}$, дістанемо векторно-матричне рівняння

$$
\frac{d \mathbf{y}(\tau, \varepsilon)}{d t}=\mathbf{D}(\tau, \varepsilon) \mathbf{y} \text {. }
$$

Тут

$$
\mathbf{D}(\tau, \varepsilon)=\left\|\begin{array}{cc}
\mathbf{A}(\tau, \varepsilon) & 0,5 \cdot \mathbf{B}(\tau, \varepsilon) \mathbf{C}^{-1}(\tau, \varepsilon) \mathbf{B}^{t}(\tau, \varepsilon) \\
2 \mathbf{W}(\tau, \varepsilon) & -\mathbf{A}^{t}(\tau, \varepsilon)
\end{array}\right\|=\sum_{k=0}^{\infty} \varepsilon^{k} \mathbf{D}_{k}(\tau)
$$

$\epsilon$ квадратною матрицею порядку $2 n$. Характер асимптотичного зображення інтеграла системи (2) істотно залежить від спектру матриці $\mathbf{D}_{0}(\tau)=\mathbf{D}(0,0)$. У $[4 ; 6]$ досліджувалися випадки стабільного спектру матриці $\mathbf{A}_{0}(\tau)$, а отже і $\mathbf{D}_{0}(\tau)$. Згідно з [6], у разі стабільного спектру останньої матриці та розв'язності матричного рівняння

$$
\mathbf{A}_{0}(\tau) Q(\tau)+Q(\tau) \mathbf{A}_{0}^{t}(\tau)-R_{0}(\tau)=0,
$$

система (2) зводиться до квазідіагональної, і асимптотичне зображення ії розв'язку отримується описаними у $[4 ; 6]$ алгоритмами. 
Припустивши, що спектр матриці $\mathbf{A}_{0}(\tau) \in$ нестабільним, розглянемо відмінності у побудові асимптоичного зображення розв'язку задачі (1) - (3). Перш за все зауважимо, що асимптотичне зображення мало рівномірний характер на досліджуваному відрізку, і був записаний за допомогою фіксованої асимптотичної шкали.

Нехай виконано одну з умов.

$4^{0}$. Принаймні одна із функцій $\lambda_{1}(\tau), \lambda_{2}(\tau), \ldots, \lambda_{n}(\tau)$ дорівнює нулю у точці $\tau=0$.

$5^{0}$. Принаймні дві функції $\lambda_{i}(\tau)$ і $\lambda_{j}(\tau)$ збігаються у точці $\tau=0$, і відрізняються в інших точках проміжку, при цьому матриця $\mathbf{A}_{0}(0)$ має просту структуру.

$6^{0}$. Принаймні дві функції $\lambda_{i}(\tau)$ і $\lambda_{j}(\tau)$ збігаються у точці $\tau=0$, і відрізняються в інших точках проміжку, при цьому матриця $\mathbf{A}_{0}(0)$ подібна жордановій клітині.

Вибір точки $\tau=0$ не $\epsilon$ принциповим, це може бути будь-яка ізольована точка проміжку, або декілька точок.

При виконанні будь-якої з умов $4^{0}-6^{0} \operatorname{det} \mathbf{A}_{0}(0)=0$, отже рівняння (8) не для будь-якої матриці $R_{0}(\tau)$ буде розв'язним, і алгоритми, розроблені у [4; 7] не дають можливості зінтегрувати задачу. Якщо виконано умову $5^{0}$, то систему (1) можна проінтегрувати багатофазовим методом, тобто записати іiі розв'язок єдиним виразом. Але це неможливо здійснити для системи (6), яку треба розв'язати при даній постановці задачі.

У роботі [6, стор. 92] для ілюстрації алгоритму розглянуто рівняння

$$
\frac{d^{2} x(\tau, \varepsilon)}{d t^{2}}+\omega^{2}(\tau, \varepsilon) x(\tau, \varepsilon)=u(\tau)
$$

що описує деяку систему зі скалярним керуванням u( $\tau)$ на проміжку $[0 ; 1]$, де

$$
\omega(\tau, \varepsilon)=\sum_{s=0}^{\infty} \varepsilon^{s} \omega_{s}(\tau), \omega_{0}(\tau)>0,0 \leq t \leq T=\frac{1}{\varepsilon}, \tau=\varepsilon t .
$$

Критерієм якості є функціонал $I(u)=\frac{1}{2} \int_{0}^{T} u^{2}(\tau) d t$.

У векторно-матричній формі досліджуване рівняння має вигляд

$$
\frac{d \mathbf{x}(\tau, \varepsilon)}{d t}=\mathbf{A}(\tau, \varepsilon) \mathbf{x}(\tau, \varepsilon)+\mathbf{B}(\tau) \mathbf{u}(\tau),
$$

де

$$
\mathbf{x}=\left(\begin{array}{l}
x_{1} \\
x_{2}
\end{array}\right), \mathbf{A}(\tau, \varepsilon)=\left(\begin{array}{cc}
0 & 1 \\
-\omega^{2}(\tau, \varepsilon) & 0
\end{array}\right), \mathbf{B}(\tau)=\mathbf{E}, \mathbf{u}(\tau)=\left(\begin{array}{c}
0 \\
u(\tau)
\end{array}\right) .
$$

Матриця $\mathbf{D}_{0}(\tau)$ системи (6) у цьому випадку має вигляд

$$
\mathbf{D}(\tau, \varepsilon)=\left(\begin{array}{cccc}
0 & 1 & 1 & 0 \\
-\omega^{2}(\tau, \varepsilon) & 0 & 0 & 1 \\
0 & 0 & 0 & \omega^{2}(\tau, \varepsilon) \\
0 & 0 & -1 & 0
\end{array}\right)
$$

Якщо $\omega(\tau, 0)>0$, як вимагається у [6], то матриця $\mathbf{D}_{0}(\tau)$ невиродженим перетворенням зводиться до діагональної

$$
\operatorname{diag}\left\{i \omega_{0}(\tau), i \omega_{0}(\tau),-i \omega_{0}(\tau),-i \omega_{0}(\tau)\right\} .
$$

Якщо $\omega(0,0)=0$, то такого перетворення не існує, а матриця $\mathbf{D}_{0}(0)$ подібна жордановій клітині із невиродженою матрицею перетворення $\mathbf{P}(0,0)$ :

$$
\mathbf{P}^{-1} \mathbf{D}(\tau, \varepsilon) \mathbf{P}=\left(\begin{array}{cccc}
0 & 1 & 0 & 0 \\
0 & 0 & 1 & 0 \\
0 & 0 & 0 & 1 \\
-\omega^{4}(\tau, \varepsilon) & 0 & -2 \omega^{2}(\tau, \varepsilon) & 0
\end{array}\right) ; \mathbf{P}(\tau, \varepsilon)=\left(\begin{array}{cccc}
1 & 0 & -1 & 0 \\
0 & 1+\omega^{2}(\tau, \varepsilon) & 1 & 0 \\
0 & -\omega^{2}(\tau, \varepsilon) & 0 & -1 \\
\omega^{2}(\tau, \varepsilon) & 0 & 1 & 0
\end{array}\right) .
$$

При побудові асимптотичного зображення розв'язку записаного рівняння використовують функції Ейрі. Для системи необхідно використати багатомасштабний метод. 
Зокрема, якщо виконано умову $2^{0}$ i $2 n \times 2 n-$ матриця $\mathbf{D}_{0}(0)$ подібна одній жордановій клітині із нульовим власним значенням, то асимптотичне зображення розв'язку за виконання додаткової умови

$$
\left.7^{0} \cdot \frac{d}{d \tau} \operatorname{det} \mathbf{D}_{0}(\tau)\right|_{\tau=0} \neq 0
$$

запишеться у вигляді

$$
\mathbf{Y}(\tau, \varepsilon)=\left\{\begin{array}{l}
\sum_{j=0}^{2 n} Z^{(j)}\left(\varepsilon^{-\frac{2 n}{2 n+1}} \xi\right) \sum_{k=0}^{\infty} \varepsilon^{k} \mathbf{U}_{j k}(\tau), \tau \in\left[0 ; L_{1} \varepsilon^{\frac{2 n}{2 n+1}}\right], \\
\sum_{k=0}^{\infty} \varepsilon^{k} \mathbf{U}_{k}(\tau) \exp \left\{\int_{0}^{t} \Lambda_{k}(s) d s\right\}, \tau \in\left[l_{2} \varepsilon^{\frac{2 n}{2 n+1}} ; L\right] .
\end{array}\right.
$$

Тут функції $Z^{(j)}(s)$ - фундаментальна система розв'язків рівняння Ейрі $Z^{(2 n)}(s)+s Z(s)=0, L_{1}$ і $l_{2}-$ сталі, що не залежать від $\varepsilon$. Перший із записаних виразів називають «внутрішнім» розвиненням розв'язку, а другий — «зовнішнім». Після визначення невідомих коефіцієнтів записаних вище формальних рядів необхідна процедура зрощування асимптотичних розвинень, якій присвячено роботу [8], в якій отримано формули звязку для «зовнішнього» та «внутрішнього» розвинень. Тільки у випадку $n=2$ формули зв'язку не потрібні можлива побудова фундаментальної матриці у вигляді єдиного виразу. Саме у записаному вище вигляді отримано результат роботи [2], де мав місце саме випадок $n=2$, тобто результат можна подати єдиним записом. В наведеному варіанті вираз для єдиного запису потребує використання розбиття одиниці.

Розглянемо спосіб розв'язування системи (6) у загальному вигляді, а для аналізу розв'язку використаємо деякі властивості системи (1) із нестабільним спектром.

Згідно з багатомасштабним методом [7], дістанемо різні вирази для асимтотичного розв'язку на проміжках

$$
\left[0 ; L_{0} \cdot \varepsilon^{r_{p}}\right],\left[l_{k} \cdot \varepsilon^{r_{p-k+1}} ; L_{k} \cdot \varepsilon^{r_{p-k}}\right],\left[l_{p} \cdot \varepsilon^{r_{1}} ; L\right] .
$$

Тут $l_{k}$ і $L_{k}$ - сталі, що не залежать від $\varepsilon ; k=1,2, \ldots, p, 0=r_{0}<r_{1}<\ldots<r_{p}$ - дійсні числа, множина яких є скінченною. Багатомасштабним методом [7] побудуємо асимптотичне зображення інтегральної матриці у вигляді

на кожному із перерахованих у (9) проміжків.

$$
\Phi\left(\xi_{k}, \varepsilon\right)=\left\|\Phi_{i j}\left(\varepsilon^{r_{p-k}} \tau, \varepsilon\right)\right\|_{i, j=1}^{2 n, n}
$$

Побудова розв'язку на всьому проміжку зміни незалежної змінної описаним методом, як зазначалося вище, потребує досить громіздкої процедури склеювання розв'язків (або узгодження асимптотичних розвинень), отриманих на вказаних проміжках. Виконавши названі процедури, отримаємо розв'язок системи (6) у формі формального ряду для інтегральної матриці

$$
\Phi(\tau, \varepsilon)=\sum_{i=0}^{p} \rho_{i}(\tau) \Phi_{i}\left(\xi_{i}, \varepsilon\right)
$$

Тут $\rho(\tau)$ - розбиття одиниці, визначене на досліджуваному проміжку. Маючи інтегральну матрицю системи (6) $\Phi(\tau, \varepsilon)=\left\|\Phi_{i j}(\tau, \varepsilon)\right\|_{i, j=1}^{2 n, n}$, можна записати вирази для невідомих векторів:

$$
\begin{aligned}
& \mathbf{x}(\tau, \varepsilon)=\left(\sum_{i=1}^{2 n} \Phi_{1 i} a_{i}, \quad \ldots, \quad \sum_{i=1}^{2 n} \Phi_{n i} a_{i}\right) ; \\
& \psi(\tau, \varepsilon)=\left(\sum_{i=1}^{2 n} \Phi_{n+1 i} a_{i}, \quad \ldots, \quad \sum_{i=1}^{2 n} \Phi_{2 n i} a_{i}\right),
\end{aligned}
$$

де $a_{i}-$ координати сталого вектора, які визначаються із крайових умов як розв'язки системи рівнянь 


$$
\left\{\begin{array}{l}
x_{0}^{i}=\sum_{j=1}^{2 n} \Phi_{i j}(0) a_{i}, \\
x_{T}^{i}=\sum_{j=1}^{2 n} \Phi_{i j}(T) a_{i} .
\end{array}\right.
$$

Методом [6] доводиться, що формальна матриця (10) є асимптотичним зображенням фундаментальної матриці $\mathrm{Y}(\tau, \varepsilon)$ системи (6) і має місце рівність $\mathrm{Y}(\tau, \varepsilon)=\Phi_{m}(\tau, \varepsilon)+O\left(\varepsilon^{m \cdot r_{1}}\right)$, де $\Phi_{m}(\tau, \varepsilon)-m$-наближення, яке дає $m$-а частинна сума ряду.

Маючи фундаментальну матрицю системи (6), повторимо міркування роботи [6, стор. 93] для побудови асимптотичного зображення синтезу оптимального керування.Знайдемо невідомі вектори $x(\tau, \varepsilon)$ та $\psi(\tau, \varepsilon)$, а отже і вектор керування $u(\tau, \varepsilon)$ з асимптотичними оцінками:

$$
\begin{gathered}
\mathbf{x}_{\mathbf{m}}(\tau, \varepsilon)=\Phi_{m,(1)}(\tau, \varepsilon) \cdot a+O\left(\varepsilon^{m \cdot r_{1}}\right), \\
\mathbf{u}_{\mathbf{m}}^{0}(\tau)=(2 C)^{-1}(\tau, \varepsilon) \cdot \mathbf{B}^{t}(\tau, \varepsilon) \cdot \Phi_{m,(2)}(\tau, \varepsilon) \cdot a+O\left(\varepsilon^{m \cdot r_{1}}\right) . \\
\Phi_{m}(\tau, \varepsilon)=\left(\Phi_{m,(1)}(\tau, \varepsilon) ; \Phi_{m(2)}(\tau, \varepsilon)\right)^{t}, \text { а також асимптотичну формулу для функціонала: } \\
\left.I_{m}=\int_{0}^{T}\left(\left(\mathbf{W}(\tau, \varepsilon) \mathbf{x}_{\mathbf{m}} \cdot \mathbf{x}_{\mathbf{m}}\right)+\mathbf{C}(\tau, \varepsilon) \mathbf{u}_{\mathbf{m}}^{0} \cdot \mathbf{u}_{\mathbf{m}}^{0}\right)\right) d t+O\left(\varepsilon^{(m-1) \cdot r_{1}}\right) \cdot
\end{gathered}
$$

Виключивши параметр $\boldsymbol{a}$ із записаних вище виразів для $\mathbf{x}_{m}$ та $\mathbf{u}_{m}$, дістанемо асимптотичне зображення для синтезу оптимального керування:

$$
\mathbf{u}_{\mathbf{m}}^{0}\left(\tau, \mathbf{x}_{\mathbf{m}}, \varepsilon\right)=(2 \mathbf{C})^{-1}(\tau, \varepsilon) \cdot \mathbf{B}^{t}(\tau, \varepsilon) \cdot \Phi_{m,(2)}(\tau, \varepsilon) \cdot \Phi_{m,(1)}^{-1}(\tau, \varepsilon) \cdot \mathbf{x}_{\mathbf{m}}+O\left(\varepsilon^{m \cdot r_{1}}\right) .
$$

У припущенні, що визначено вектор керування, розглянемо систему (1), яка стане неоднорідною системою

$$
\frac{d \mathbf{x}(\tau, \varepsilon)}{d t}=\mathbf{A}(\tau, \varepsilon) \mathbf{x}(\tau, \varepsilon)+\mathbf{f}(\tau)
$$

Якщо остання система має в точці $\tau_{1}$ нестабільність у спектрі таку, що принаймні одне власне значення $\lambda\left(\tau_{1}\right)=0$, то $\operatorname{det} \mathbf{A}\left(\tau_{1}, 0\right)=0$, і розв'язок (а не тільки його асимптотичне зображення) цієї системи при $\mathbf{f}\left(\tau_{1}\right) \neq 0$ матиме необмеженість при $\varepsilon \rightarrow 0$, що може бути небажаним у багатьох реальних системах. Зокрема, якщо $\mathbf{A}\left(\tau_{1}, 0\right)$ подібна жордановій клітці порядку $n$, то $|\mathbf{x}(\tau, \varepsilon)|=O\left(\varepsilon^{-\frac{1}{n}}\right)$ навіть за виконання умови стійкості. При наявності точок поворот таких, що $\operatorname{det} A\left(\tau_{1}, 0\right) \neq 0$, розв'язок залишатиметься обмеженим за виконання умови стійкості. Отже, забезпечення умови обмеженості розв'язку можливе при $\mathbf{f}\left(\tau_{1}\right)=0$. Це можливо або при виконанні рівності $\mathbf{B}\left(\tau_{1}, 0\right)=0$ або $\mathbf{u}\left(\tau_{1}, 0\right)=0$. Іншими словами, керування має бути рівним нулю в момент проходження системою точки повороту.

\section{Висновки}

Нестабільність у спектрі головної матриці системи призводить до того, що побудова асимптотичного зображення розв'язку є значно складнішим, і потребує громіздких процедур при конструюванні фундаментальної матриці. Проте це відноситься до суто математичної частини дослідження. Реальність і фізичний зміст точок нестабільності потребує аналізу для кожної конкретної системи керування.

\section{Список використаної літератури}

1. Абгарян К. А. Матричные и асимптотические методы в теории линейных систем. Москва: Наука, 1973. $431 \mathrm{c}$.

2. Leifura V. N. On One Problem of Automatic Control with Turning Points. Symmetry in Nonlinear Mathematical Physics : Proccedings of the Second International Conference. Kyiv, 1997. V. 2. P. 488-491.

3. Ломов С. А. Введение в общую теорию сингулярных возмущений. Москва : Наука, 1981. $400 \mathrm{c}$. 
4. Моисеев Н. Н. Асимптотические методы нелинейной механики. 2-е изд., перераб. Москва : Наука, 1981. 400 c.

5. Самойленко А. М., Шкіль М. І., Яковець В. П. Лінійні системи диференціальних рівнянь 3 виродженнями. Київ : Вища шк., 2000. 294 с.

6. Шкиль Н. И., Вороной А. Н., Лейфура В. Н. Асимптотические методы в дифференциальных и интегро-дифференциальных уравнениях. Киев : Выща шк., 1985. 248 с.

7. Wasow W. Linear Turning Point Theory. New York : Acad. Press, 1985. 246 p.

8. Wasow W. The central connection problem at turning points of linear differential equations. Commentarii Helv. 1971, v. 46. P. 65-86.

\section{ASYMPTOTIC SOLUTION OF THE OPTIMAL CONTROL PROBLEM NON-STATIONARY SYSTEMS Rashevs'kyi M.}

\section{Abstract}

Models of non-stationary automatic control systems are differential equations with variable coefficients. Such equations do not integrate in quadratures in the general case. Asymptotic methods are methods of approximate integration of differential equations with variable coefficients.

In the article the non-stationary automatic control system with slowly variable parameters is considered. To study this system it is necessary to construct an asymptotic representation of its solution. In the theory of asymptotic integration exist a problem to construction of the asymptotic solution of a system in the presence of a turning point. Special methods have been developed to construct a solution to such systems. The most common methods is the method of reference equations, the method of the Maslov's canonical operator, the multiphase Kucherenko method, the method of W. Wasow.

The purpose of the article is to construct an asymptotic solution of a linear system of differential equations with available a turning point.

In this article the asymptotic representation of the solution of the optimal correction problem is constructed. The case of nonstability spectrum of the main matrix and the available of turning points are investigated. Application of the Pontryagin maximum principle to the problem leads to a system with slowly varying coefficients and an nonstable spectrum. Construction of a formal solution of the main system with turning points in the form of a single expression in some cases is possible. The system formed in the process of solving the problem of optimal correction does not allow the mentioned construction. A multiscale method was used to solve this system of equations. Asymptotic estimates for the constructed approximations are given. The studied problem has practical applications in technical and economic systems, in particular in the calculation of the correction of the orbits of artificial satellites.

Further research may be aimed at finding a unified approach to solving such problems and to ascertain the physical meaning of the turning point in specific systems of automatic control.

\section{References}

[1] Abgaryan, K.A. (1979). Matrichniye $i$ asimptoticheskiye metody v teorii lineinyh system [Matrix and asymptotic methods in the theory of linear systems]. Moscow: Nauka [in Russian].

[2] Leifura, V.N. (1997). On One Problem of Automatic Control with Turning Points. Proceeding of the 2nd International Conference «Symmetry in Nonlinear Mathematical Physics», 2, (pp. 488491). Kyiv: Institute of Mathematics. Retrieved from https://www.slac.stanford.edu/econf/C9707077/papers/art68.pdf

[3] Lomov, S.A. (1981). Vvedenie v obshchuyu teoriyu singulyarnyh vozmushchenij [Introduction to the general theory of singular perturbations]. Moscow: Nauka [in Russian]. 
[4] Moiseev, N.N. (1981) Asimptoticheskiye metody nelineinoj mehaniki [Asymptotic methods of nonlinear mechanics] (2 ${ }^{\text {nd }}$ ed.,rev.). Moscow: Nauka [in Russian].

[5] Samoilenko, A.M., Shkil', M.I., \& Yakovets, V.P. (2000). Linijni Systemy dyferentsial'nyh rivnyan' $z$ vyrodzhennyamy [Linear systems of differential equations with degenerations]. Kyiv: Naukova Dumka [in Ukrainian].

[6] Shkil', M.I., Voronoj, A.N., \& Leifura, V.N. (1985). Asimptoticheskiye metody v differentsyal'nyh $i$ integro-differentsyal'nyh uravneniyah [Asymptotic methods in differential and integrodifferential equations]. Kyiv: Vyshcha Shkola [in Russian].

[7] Wasow, W. (1966). On a Turning Point Problems for Systems with Almost Diagonal Coefficient Matrix. Funkcialaj Ekvacioj, (8(3)), 143-171.

[8] Wasow, W. (1971). The central connection problem at turning points of linear differential equations. Commentarii Mathematici Helvetici. (46), 65-86. DOI

https://doi.org/10.1007/BF02566828 